$$
\begin{gathered}
507 \mathrm{OS} \\
00 \mathrm{TI}
\end{gathered}
$$

TITLE: THE GEM SILICON TRACKING SYSTEM

AUTHOR(S): Geoffrey B. Mills

SUBMITTED TO
Presented at the International Symposium on Development and Application of Semiconductor Tracking Detectors, on May 22-24, 1993, Hiroshima, Japan




\title{
The GEM Silicon Tracking System
}

\author{
Geoffrey B. Mills \\ Physics Division \\ Los Alamos National Laboratory
}

\begin{abstract}
The GEM Collaboration has produced a baseline design for the GEM detector. The baseline design of the GEM Silicon Tracking System (STS) is discussed in this article. Mechanical and electrical engineering progress on the GEM STS is described. Results from simulations of detector performance and the implications on engineering issues are described.
\end{abstract}




\section{Introduction}

The design of the GEM Silicon Tracking System (STS) has evolved to a conceptual design over the last two years. This article discusses the status of the design at the point of the GEM Collabcration's (Gamma Electron Muon) technical design report. The physics goals of the STS are to: define the vertex for the physics event of interest; separate electrons and gammas; determine the electron sign up to 600 $\mathrm{GeV} / \mathrm{c}$; reconstruct the charged tracks in each event; find secondary vertices; and provide background rejection by measuring the momentum of each track. The GEM detector is shown in Figure 1, and the Central Tracker is shown in Figure 2.

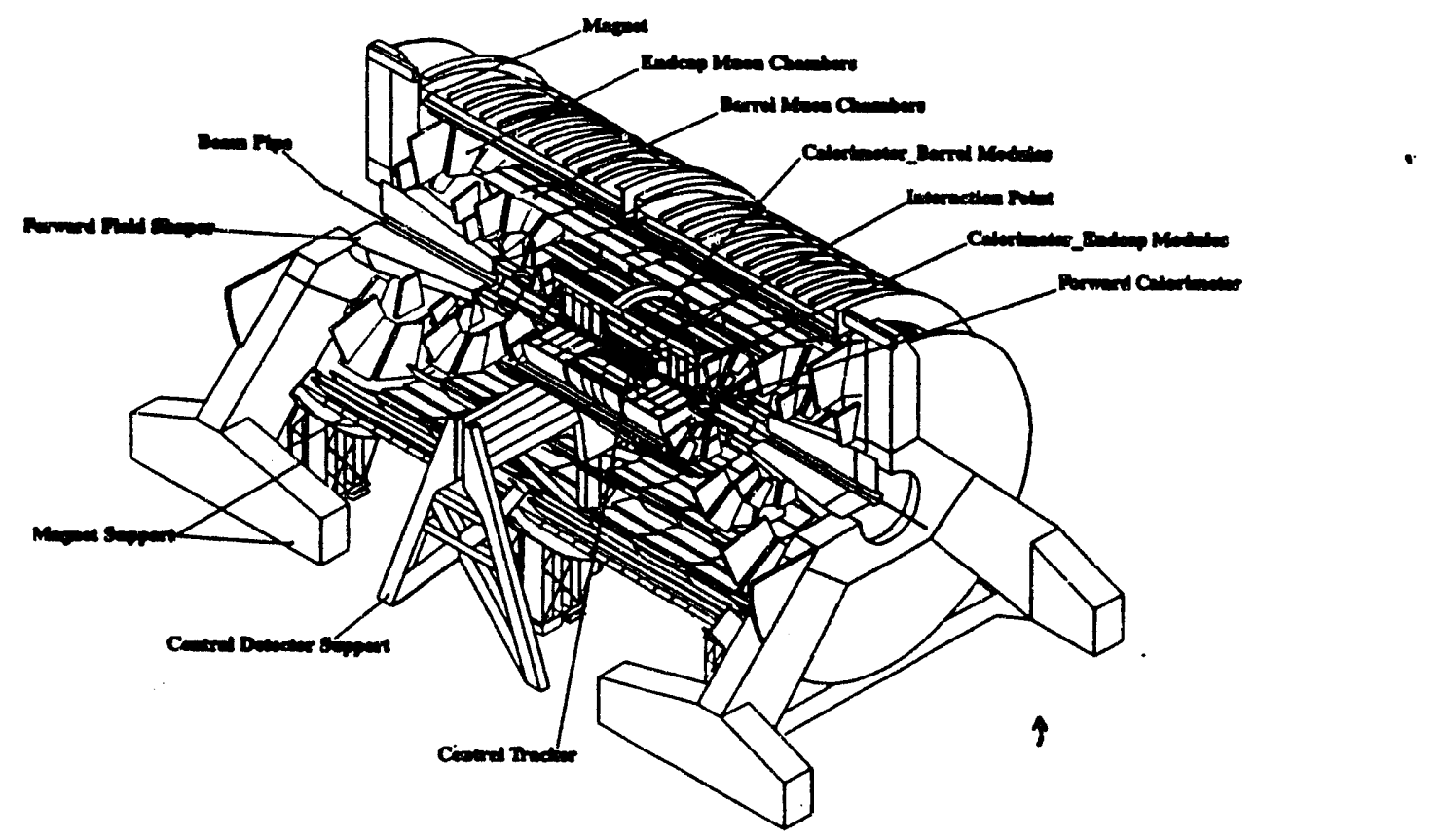

Figure 1 Perspective view of the GEM detector

The Central Tracker is at the center of the GEM detector and is the subsystem closest to the interaction point. Because of the close proximity to the beam and the harsh radiation environments, the Central Tracker must be designed to survive high doses of hadron raviation and remain stable. The Central Tracker has been divided into two subsystems, an inner Silicon Tracking System and an outer Interpolating Pad Chamber (IPC). The past two years have seen work on the R\&D necessary to define solutions to this difficult design problem and the completion of a conceptual design.

The mechanical design of the STS entails: the support structure; the silicon ladders; the cooling system; the containment vessel; and the alignment system. Designs are progressing on the radiation hardened front-end electronics that will provide the readout for the silicon strips. In addition, work is taking place on the GEANT simulations of the silicon tracker and the overall pattem recognition. 


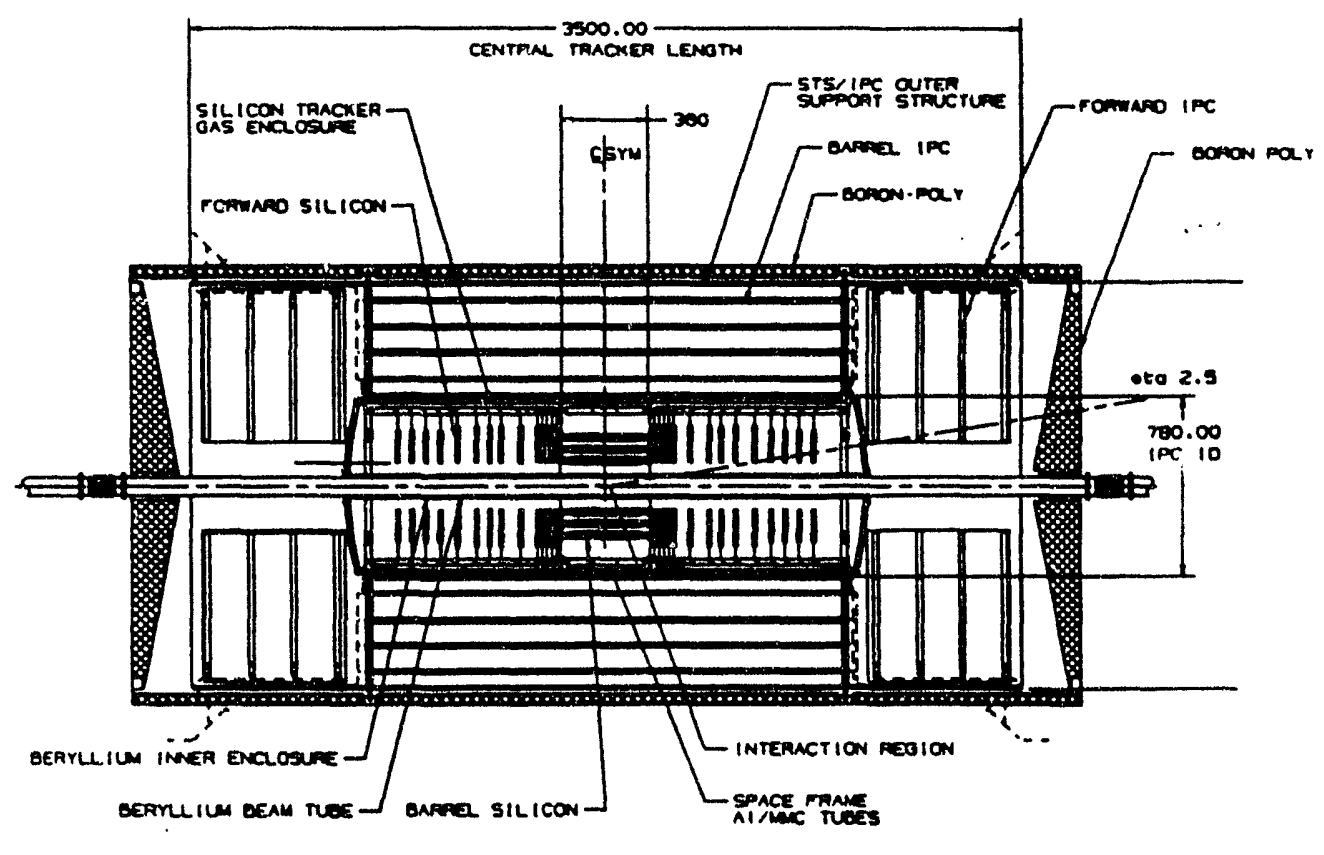

Figure 2 The GEM Central Tracker

\section{GEM STS Mechanical Systems}

The design parameters for the GEM Silicon Tracking System (STS) are set by physics requirements of resolution, occupancy, and speed. The critical performance parameter of the tracker is the spatial resolution of 15 microns per layer in the azimuthal direction derived jointly from the momentum resolution and the impact parameter resolution requirements. The resolution requirements translates into a stability requirement of $10 \mu \mathrm{m}$ for the mechanical structures. Physics simulations show that $10 \mu \mathrm{m}$ stability is needed to preserve the ability to find secondary vertices. To achieve the highest efficiency for detecting electrons and photons in GEM, we must minimize electron bremsstrahlung and photon conversions in the material of the tracker. Therefore, it is important to limit the radiation length in the silicon system. A conceptual design that meets all of the physics requirements has been developed.

A cut-away view of the Silicon Tracking System is shown in Figure 3 It consists of a barrel region 


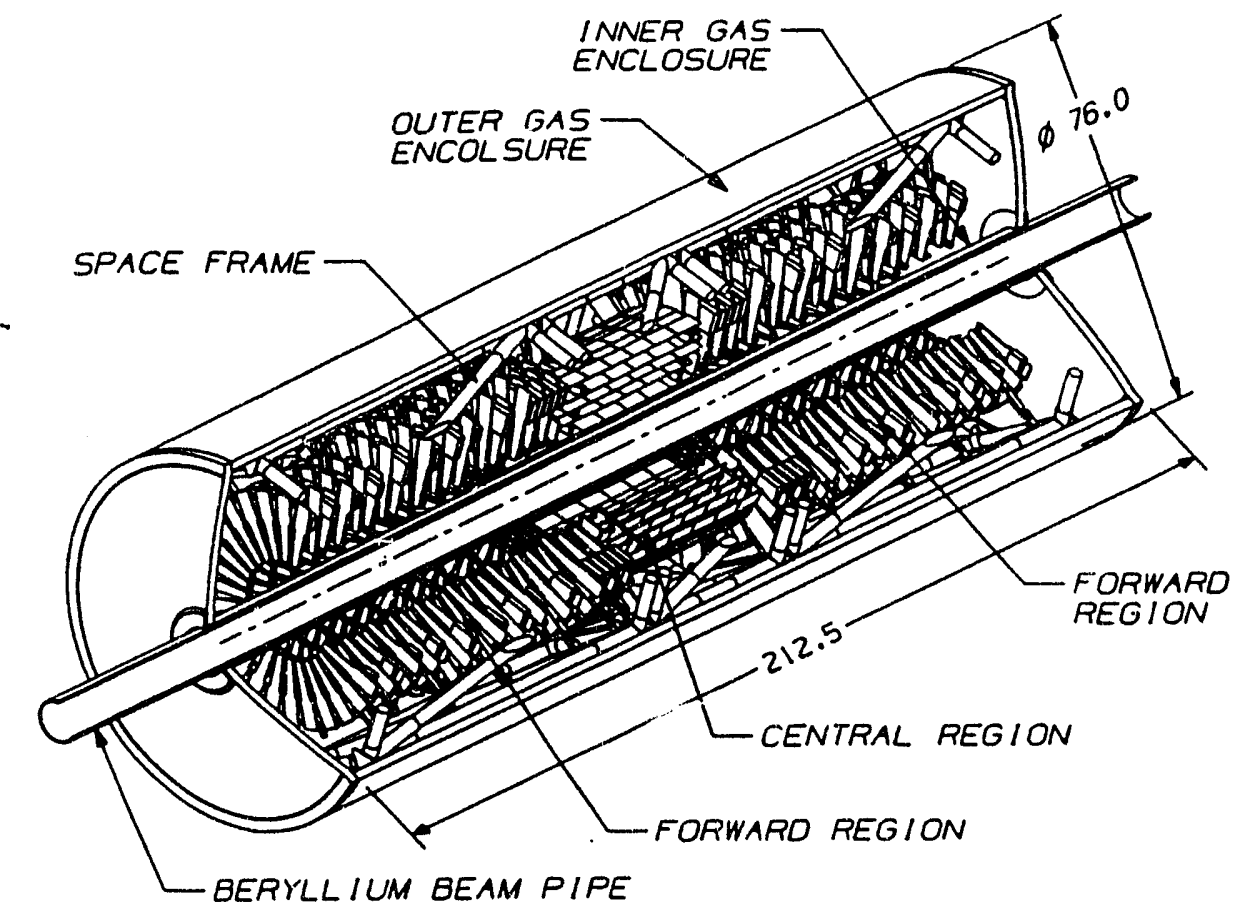

Figure 3 Cut-Away of the Silicon Tracking System

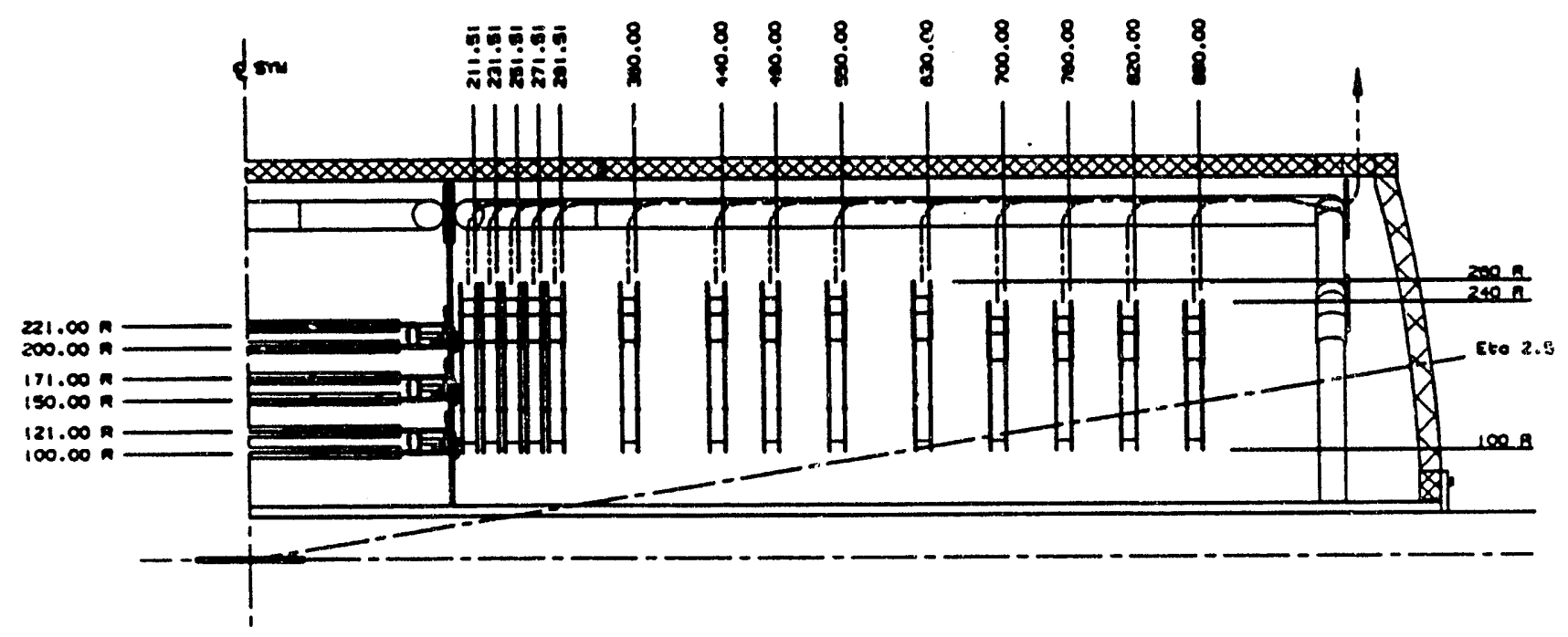

Figure 4 Silicon detector barrel and forward modules 
and two forward regions. The barrel region consists of six layers of silicon ladder assemblies, extending $18 \mathrm{~cm}$ axialiy on each side of the interaction region and at radii between $10 \mathrm{~cm}$ and $22 \mathrm{~cm}$. Each forward region contains fourteen disks of silicon ladders. The barrel and forward modules are shown in Figure 4. The visible support structure and enclosures are made of advanced materials, such as metal matrix coinposites and graphite/cyanate-ester composites. The cooling system has been designed to remove the $6.8 \mathrm{~kW}$ heat load of the electronics efficiently, by using an evaporative wick located close to the electronics. We have carefully simulated this design to determine that the placement of the forward disks preserves the geometrical efficiency when the $5 \mathrm{~cm}$ uncertainty of the interaction is included. In addition, we used the physics simulation code to determine the radiation length of the silicon tracker, and its effect on the detection efficiency for electrons and photons. Similar calculations will have to be done as the design efforts proceed beyond the conceptual phase.

\section{GEM STS Front-End Electronics Systems}

The GEM Silicon Tracking System (STS) front-end electronics requires the application of high speed (60MHz), low power ( $<2 \mathrm{~mW} /$ channel), low noise $(<2500$ electrons), low mass, VLSI electronics in high radiation environment (up to $10^{14} \mathrm{p} / \mathrm{cm}^{2}$ total fluence). These severe requirements are unprecedented in any electronic development to this day. Many advanced developments along these lines have occurred in space science, where low power, high reliability, and radiation hardness are also key issues.

The design of the GEM STS front-end system has evolved over the last two years. There are approximately 2.5 million strips in the silicon system, each requiring an individual amplifier and readout chain. The Silicon Tracker System electronics architecture is shown in Figure 5. The purpose of the system is to take raw hit information, perform zero suppression, and transmit the zero suppressed data to the off-detector electronics. The readout system for the silicon ladder resides physically on a $3 \mathrm{~cm} \times 6 \mathrm{~cm} \times 700 \mu \mathrm{m}$ Multi-Chip Module (MCM), which is bonded to the silicon ladder and provides the readout for 1280 silicon strips. A total of 1942 MCM's is needed to complete the system. The MCM consists of ten pairs of custom integrated circuits (bipolar and CMOS-1), two custom CMOS-2 chips common to all ten pairs, and a pair of LED optical transmitters. Each pair of custom integrated circuits handles 128 channels of the strip detectors. The two controller chips, called CMOS-2A and CMOS-2D, serve all ten CMOS- 1 chips by controlling data flow and by providing the housekeeping function for the MCM. All components must be radiation hardened, highly reliable, and low power.

The signal path begins at the analog bipolar chip, where the strip detector signal is amplified and discriminated. The discriminated signal is then fed to a data driven buffer which resides on CMOS-1, where it is stored until a level 1 trigger accept signal arrives. Zero suppression circuitry, also located on 


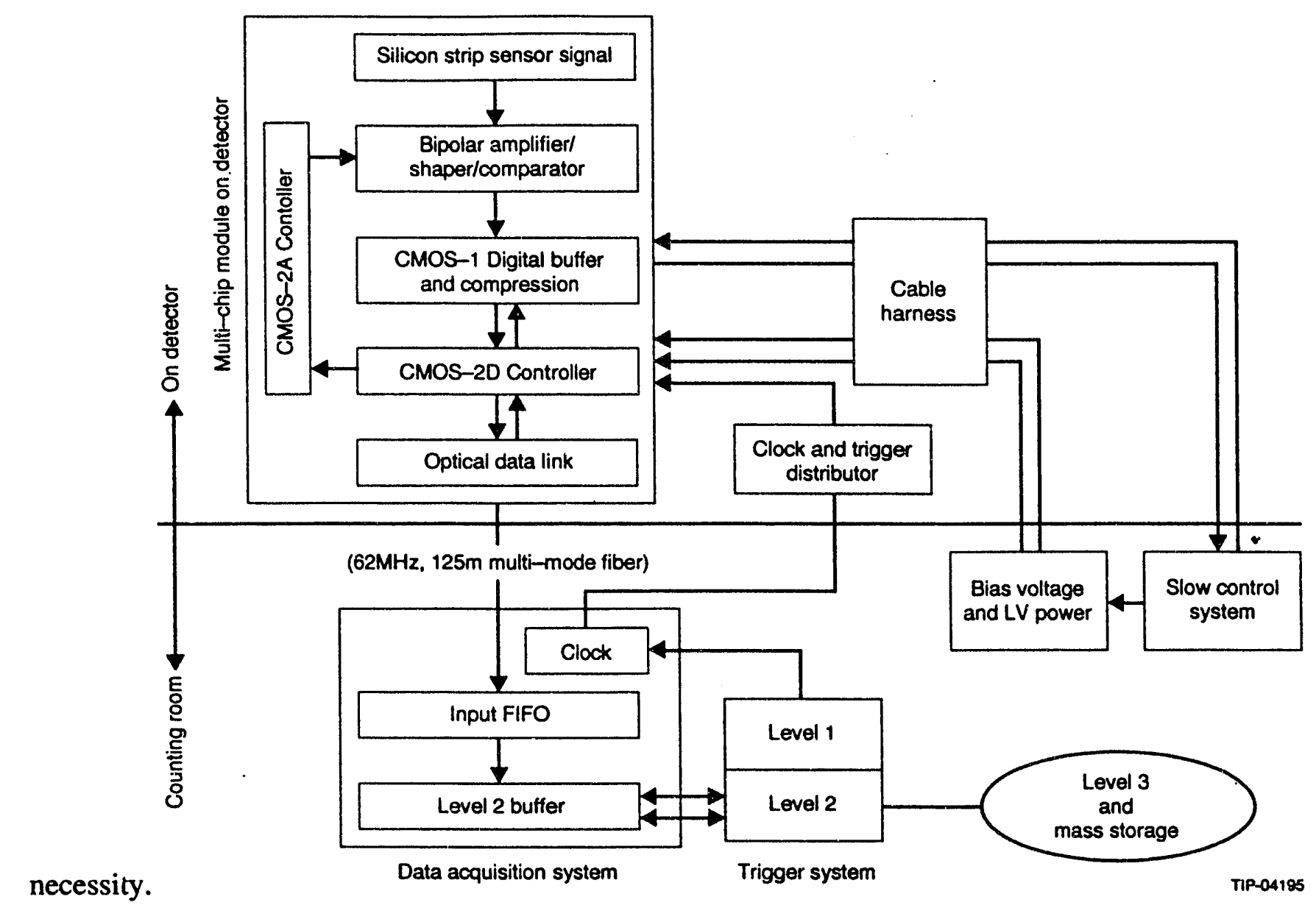

Figure 5 Silicon tracker electronics system architecture

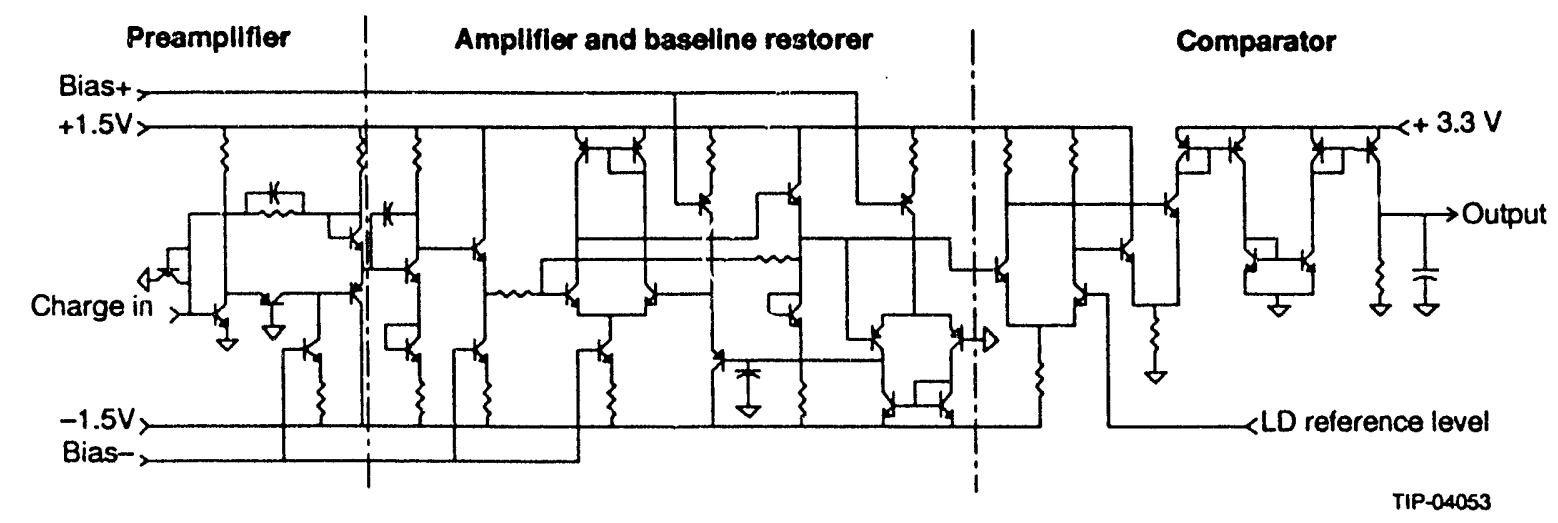

Figure 6 Silicon Tracker Bipo'.ar ASIC

CMOS-1, then compresses the data and ships it via CMOS-2 to a LED fiber-optic data link which terminates in the counting house electronics. Figure 6 shows the prototype bipolar analog chip. It 
includes the preamplifier, amplifier, shaper and baseline restorer, and level discriminator. Simulations show that this circuit meets the design criteria. A semi-custom ASIC was fabricated to verify the validity of the simulation results, and the fabricated IC agreed in all aspects with the simulated results. One goal of the front-end design is to preserve the beam crossing information. This has been analyzed by taking the pulse waveform from the analog amplifier and folding it with the expected Landau fluctuations to determine the time walk from the discriminator section. We find that greater than 98 percent of the signals fall within the $16 \mathrm{~ns}$ time window.

The noise performance of the amplifier has been simulated for a number of source impedance. Table 1 shows the results of those simulations as a function of inter-strip capacitance and strip linear resistance. These simulations have been verified by measurement at several reference points. In these simulations it has been essential to put in the actual strip line characteristics. This is because the system of coupled strip lines which forms an RC network which degrades the signal that the amplifier receives, and hence degrades the equivalent charge noise which one measures (ENC). The table below contains all of these effects in it. The $18 \mathrm{~cm}$ strip length should achieve the goal of $1.1 \mathrm{pF} / \mathrm{cm}$ with $20 \Omega / \mathrm{cm}$.

Table 1. ENC as a Function of Linear Strip Resistance and Capacitance

\begin{tabular}{|c|c|c|c|}
\hline \multicolumn{4}{|c|}{ Equivalent Noise for Far(Near) End Charge Injection for $18 \mathrm{~cm}$ strips } \\
\hline \multirow{2}{*}{\multicolumn{2}{|c|}{$\begin{array}{c}\text { Noise vs Capacitance } \\
\qquad(20 \Omega / \mathrm{cm})\end{array}$}} & \multirow{2}{*}{\multicolumn{2}{|c|}{$\begin{array}{l}\text { Noise vs Resistance } \\
(1.0 \mathrm{pF} / \mathrm{cm})\end{array}$}} \\
\hline & & & \\
\hline $0.6 \mathrm{pF} / \mathrm{cm}$ & $1948(1916)$ & $10 \Omega / \mathrm{cm}$ & 2191(2180) \\
\hline $0.8 \mathrm{p} 5 / \mathrm{cm}$ & $2122(2092)$ & $20 \Omega / \mathrm{cm}$ & $2276(2233)$ \\
\hline $1.0 \mathrm{pF} / \mathrm{cm}$ & $2276(2233)$ & $30 \Omega / \mathrm{cm}$ & $2356(2272)$ \\
\hline $1.2 \mathrm{pF} / \mathrm{cm}$ & $2432(2371)$ & $40 \Omega / \mathrm{cm}$ & 2431(2303) \\
\hline $1.4 \mathrm{pF} / \mathrm{cm}$ & $2664(2579)$ & $50 \Omega / \mathrm{cm}$ & 2501(2327) \\
\hline
\end{tabular}

\section{GEM STS Performance Simulations}

A Monte Carlo code based on GEANT has been developed to study issues relating to tracking for the GEM detector. The code includes a detailed description of the silicon tracker and the IPC system. The code is used to help in the optimization of the mechanical and electronic design as well as the study of the capabilities of the Central Tracker for interesting physics processes. This Monte Carlo code is the basis for simulations performed by the Central Tracker group. The results of some of the sirnulations are shown in Figures 7-9. Figure 7 shows the momentum resolution at 90 degrees for the Central Tracker as a function of momentum. The multiple scattering is negligible for the higher momenta, and we see 
that the measurement error is $\Delta \mathrm{p}_{t} / \mathrm{p}^{-} .001[\mathrm{GeV} / \mathrm{c}]^{-1} \cdot \mathrm{p}_{t}$, which is consistent with the physics requirements. Figures 8 and 9 show the impact parameter resolution as a function of momentum and pseudorapidity, respectively. Again, the design criterion of $30 \mu \mathrm{m}$ is satisfied.

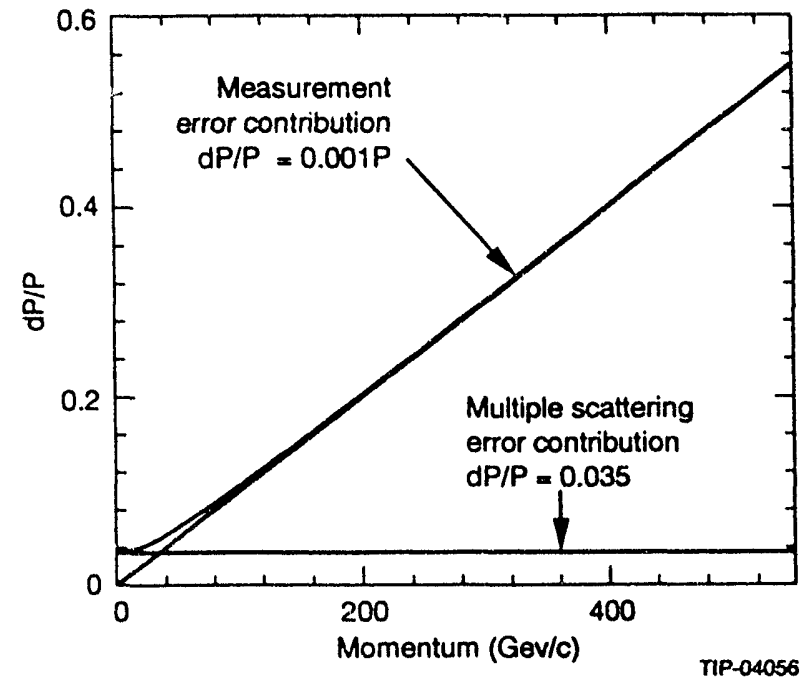

Figure 7 Momentum resolution vs. momentum

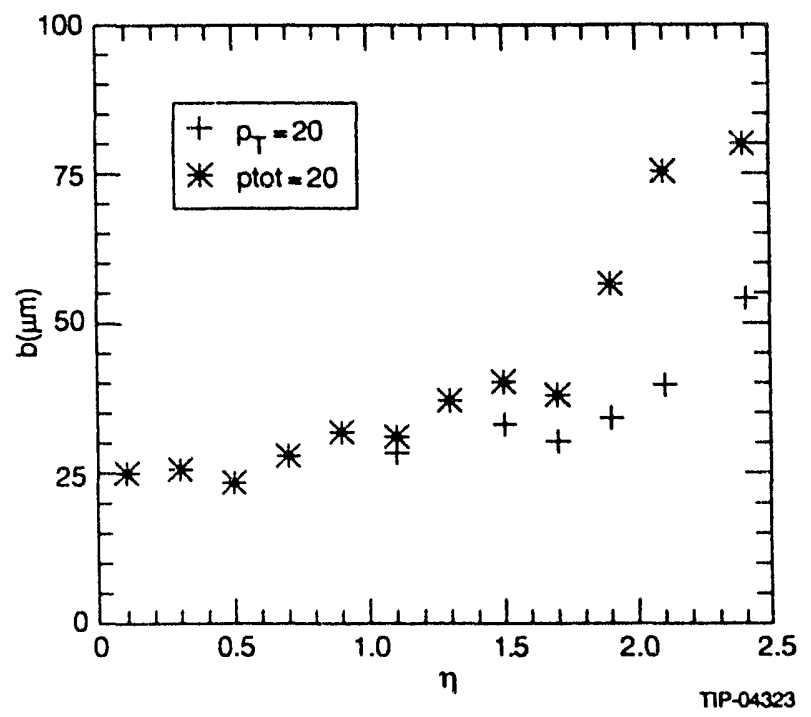

Figure 9 Impact parameter resolution vs. pseudorapidity.

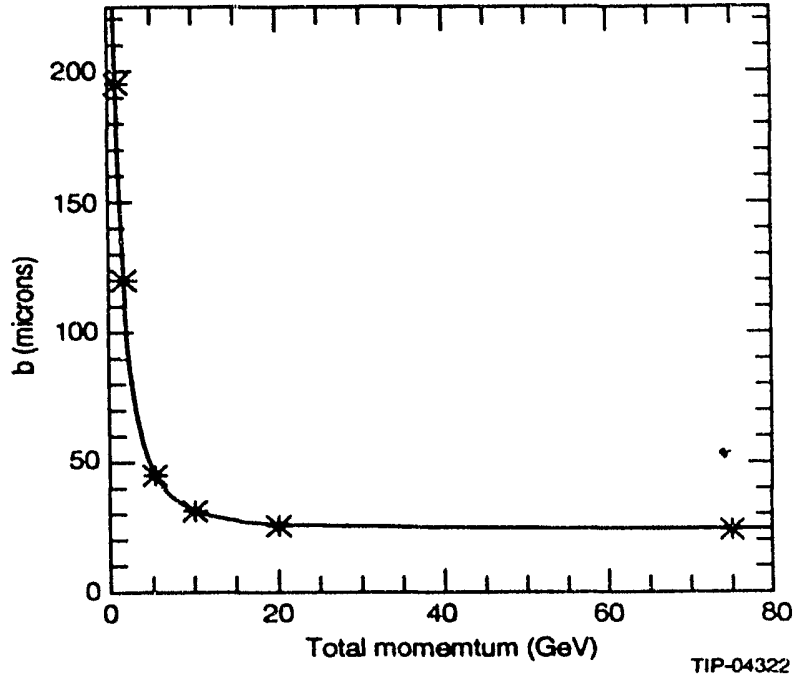

Figure 8 Impact parameter resolution vs. momentum

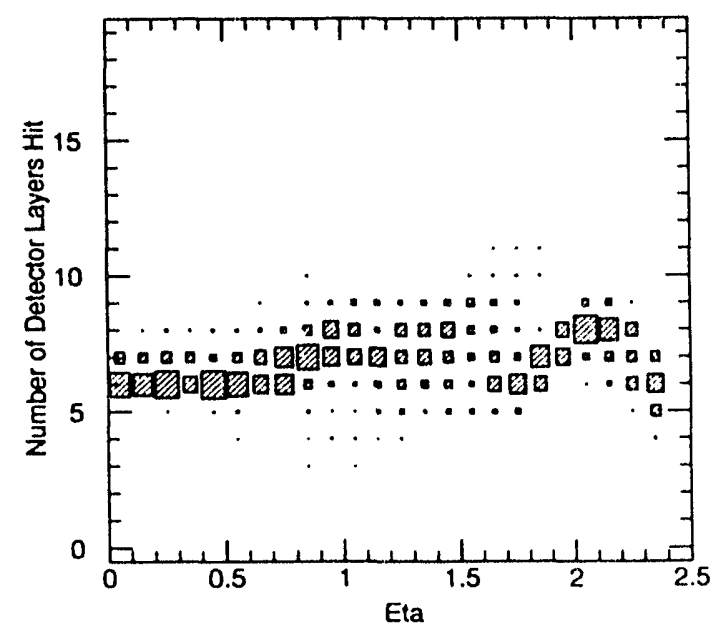

Figure 10 STS coverage: number of planes hit vs. pseudorapidity. 
The code has been used to optimize the electronics and mechanical design. Studies of the geometrical efficiency are used to understand the proper placement of the forward disks. Simulated electronic noise sources enable the understanding of the limits they place on the noise figure of the bipolar amplifier and the silicon detector strip lengths. The simulation also enables the understanding of the contribution each component makes to the total radiation length of the detector. Figures 9- 12 illustrate these important aspects of our simulation studies. Figure 10 displays the number of detector layers hit by an energetic muon as a function of pseudorapidity for the Silicon Tracking System. This includes vertex smearing, which complicates any naive placement of layers. Previous studies have shown that 6 layers give good reconstruction efficiencies.

The forward disks are placed so that at least 6 layers are hit most of the time, but not significantly more than 6. Figure 11 shows a study of the efficiency as a function of the threshold-to-noise. We found that a threshold-to-noise of $3 / 1$ along with an equivalent noise charge of less than 3000 were required to maintain good efficiency. Figure 12 shows the radiation length contribution from various detector components. This type of study is used to direct our attention to areas in the detector where a combination of components has contributed to unacceptably large radiation lengths.

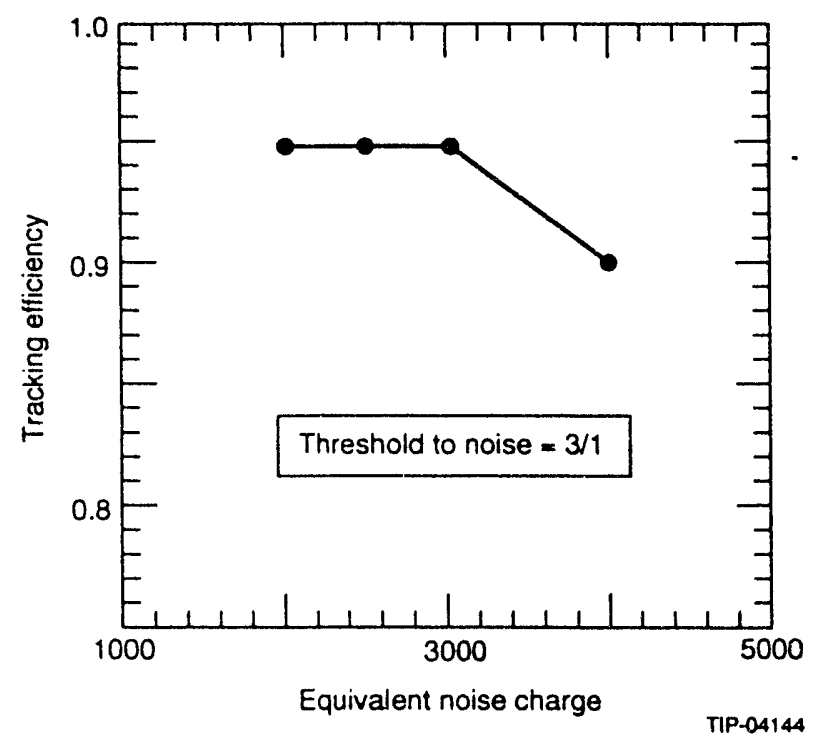

Figure 11 Tracking efficiency vs. equivalent noise charge in the analog electronics, at fixed threshold-to-noise ratio.

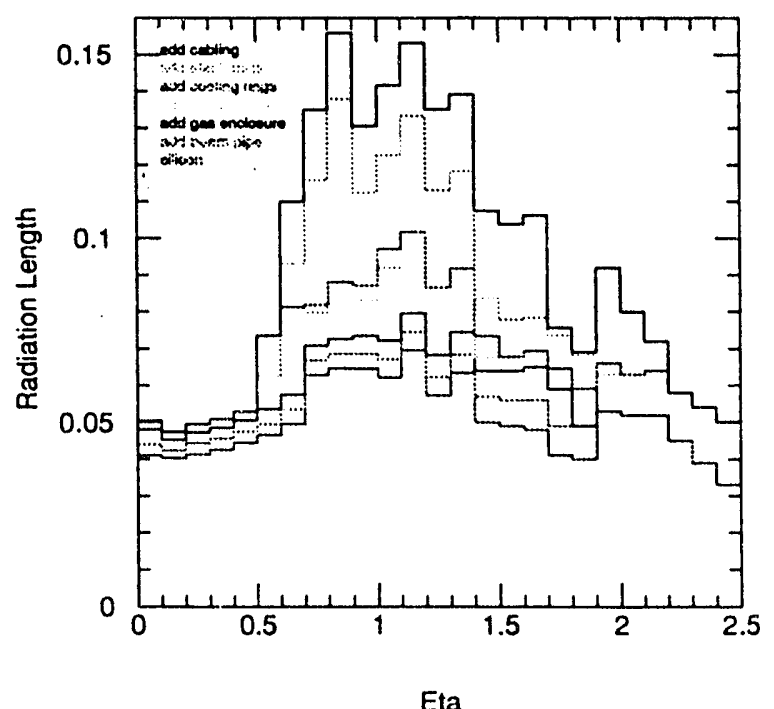

Figure 12 Radiation Length of material in the GEM STS vs. pseudorapidity.

The track finding efficiency of the detector depends upon the geometric efficiency, the detector efficiency, track density, the dead time of the detectors, the number of layers and the placement of the layers in the detector, and the pattern recognition algorithm itself. The current pattern recognition 


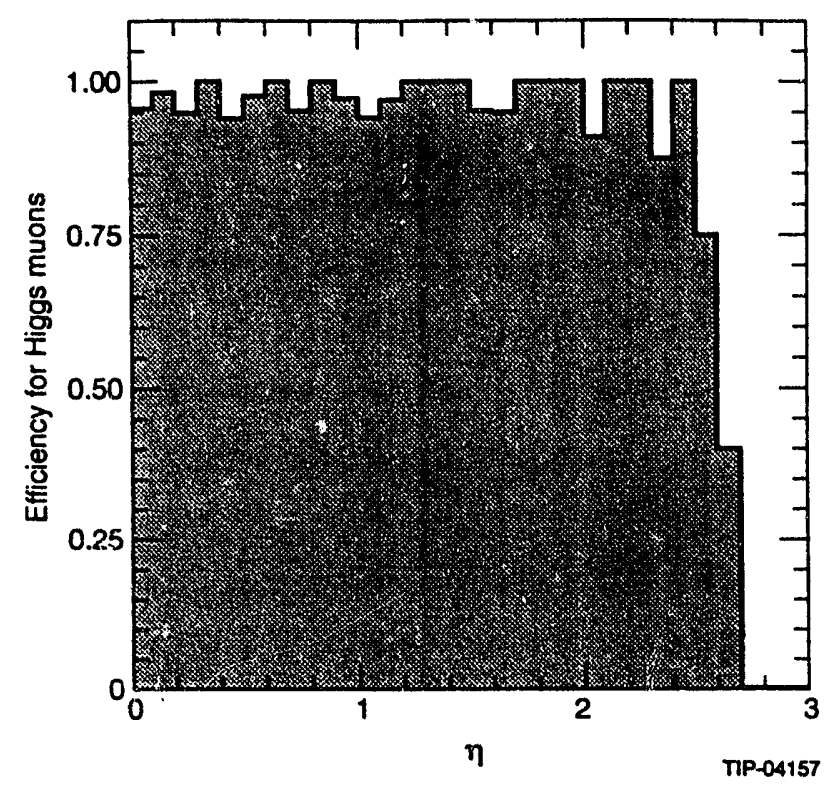

Figure 13 Efficiency for finding muons from Higgs decay vs. pseudorapidity.

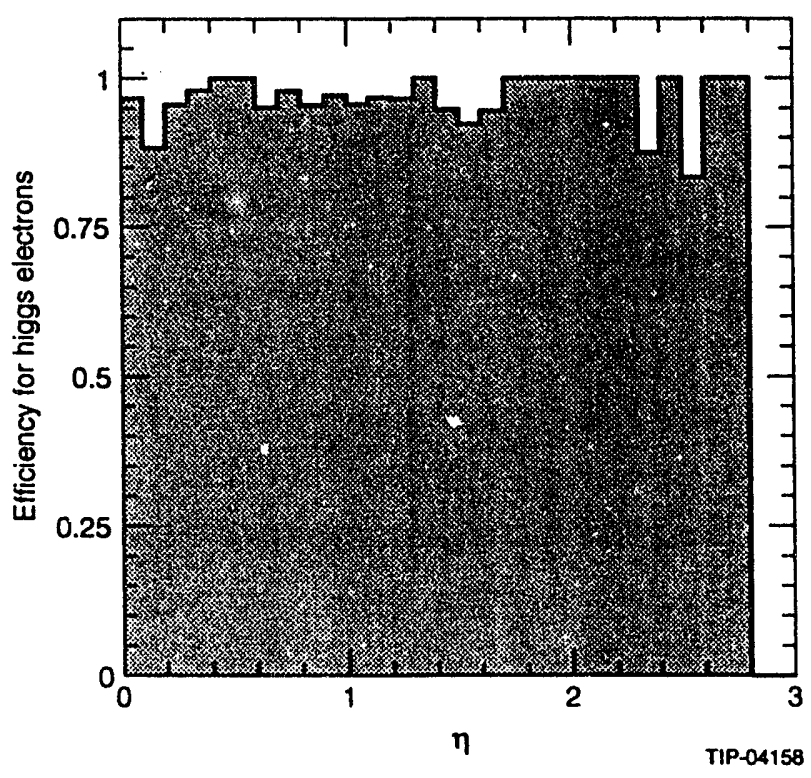

Figure 14 Efficiency for finding electrons from Higgs decay vs. pseudorapidity.

algorithm builds vector trees originating on an inner layer of the tracker. The tree is searched for the set of vectors with the best $\chi^{2}$. This track candidate is then fitted to a helix to give final track parameters.

Figure 13 presents the efficiency for finding muons from Higgs decay as a function of pseudorapidity. Figure 14 presents the efficiency for finding electrons from Higgs decay. In both cases 
the efficiency is $97 \%$. With this comprehensive code we have initiated physics studies for the Central Tracker. Other important areas of study which have been invesiigated are: the efficiency for identifying b-jets from all other jets, important for top quark physics and B meson physics; and the study of the detection efficiency for photons from Higgs decay together with the rejection efficiency for electrons, a serious background in the decay mode $H^{0} \rightarrow \gamma \gamma$.

\section{Summary}

A baseline design for the GEM silicon tracker is a 2.5 million channel system with a maximum strip length of $18 \mathrm{~cm}$ in the barrel region. The mechanical layout goal of having at least 6 space point hits per track in the pseudorapidity range \pm 2.5 has been accomplished. The radiation length requirements, critical to tracker performance, appear to be achievable. Noise performance in a bipolar amplifier sufficient to meet GEM tracking needs has been demonstrated. The overall performance of the silicon tracker is sufficient to meet the baseline goals of the GEM experiment. 

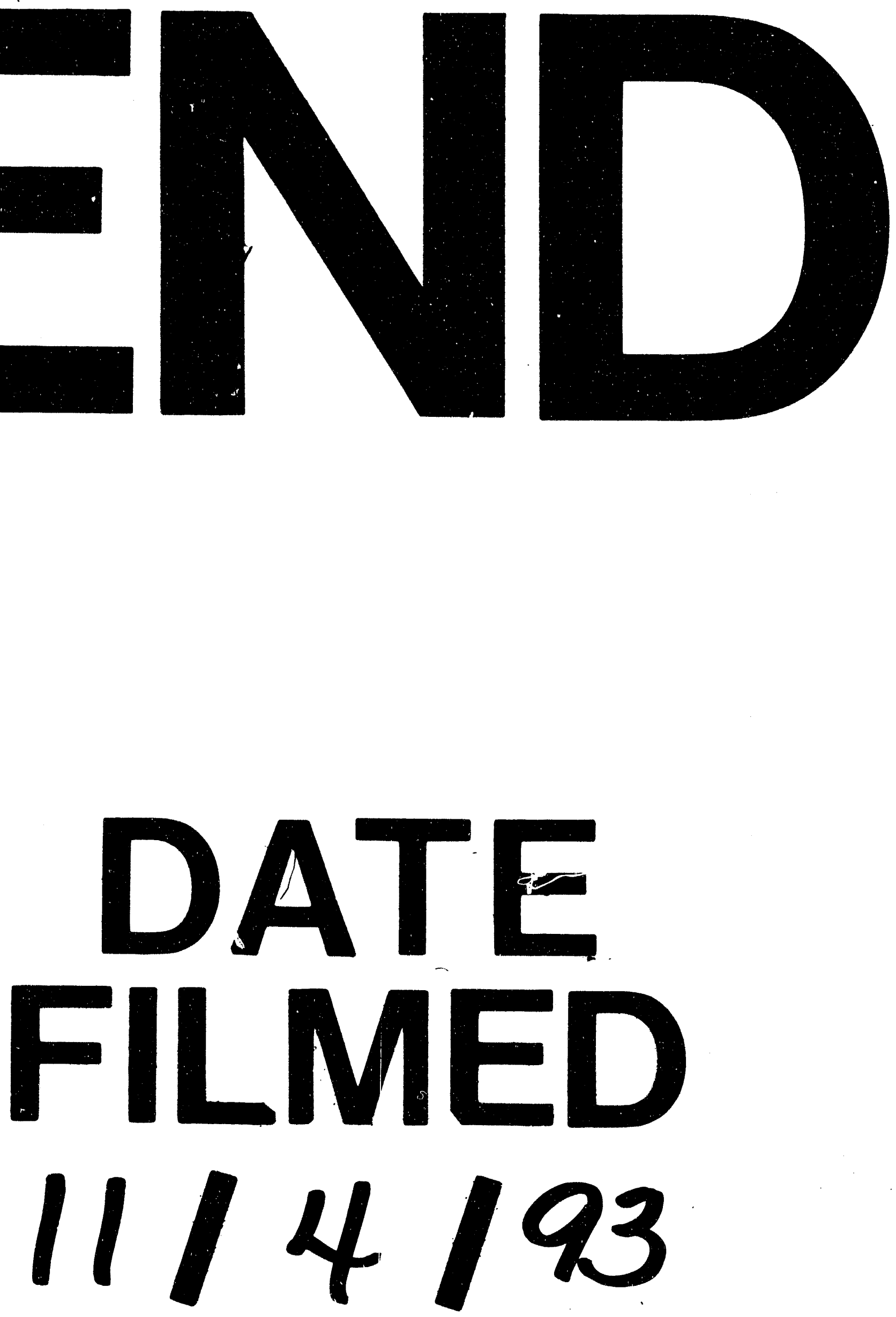
